Louisiana State University

LSU Digital Commons

Faculty Publications

Department of Physics \& Astronomy

$1-10-2006$

\title{
Optical light curve and cooling break of GRB 050502A
}

\author{
S. A. Yost \\ University of Michigan, Ann Arbor \\ K. Alatalo \\ University of Michigan, Ann Arbor \\ E. S. Rykoff \\ University of Michigan, Ann Arbor \\ F. Aharonian \\ Max Planck Institute for Nuclear Physics \\ C. W. Akerlof \\ University of Michigan, Ann Arbor
}

See next page for additional authors

Follow this and additional works at: https://digitalcommons.Isu.edu/physics_astronomy_pubs

\section{Recommended Citation}

Yost, S., Alatalo, K., Rykoff, E., Aharonian, F., Akerlof, C., Ashley, M., Blake, C., Bloom, J., Boettcher, M., Falco, E., Göğüş, E., Güver, T., Halpern, J., Horns, D., Joshi, M., Kiziloğlu, Ü., McKay, T., Mirabal, N., Özel, M., Phillips, A., Quimby, R., Rujopakarn, W., Schaefer, B., Shields, J., Skrutskie, M., Smith, D., Starr, D., Swan, H., Szentgyorgyi, A., Vestrand, W., Wheeler, J., \& Wren, J. (2006). Optical light curve and cooling break of GRB 050502A. Astrophysical Journal, 636 (2 I), 959-966. https://doi.org/10.1086/498134

This Article is brought to you for free and open access by the Department of Physics \& Astronomy at LSU Digital Commons. It has been accepted for inclusion in Faculty Publications by an authorized administrator of LSU Digital Commons. For more information, please contact ir@lsu.edu. 


\section{Authors}

S. A. Yost, K. Alatalo, E. S. Rykoff, F. Aharonian, C. W. Akerlof, M. C.B. Ashley, C. H. Blake, J. S. Bloom, M. Boettcher, E. E. Falco, E. Göğüş, T. Güver, J. P. Halpern, D. Horns, M. Joshi, Ü Kiziloğlu, T. A. McKay, N.

Mirabal, M. Özel, A. Phillips, R. M. Quimby, W. Rujopakarn, B. E. Schaefer, J. C. Shields, M. Skrutskie, D. A.

Smith, D. L. Starr, H. F. Swan, A. Szentgyorgyi, W. T. Vestrand, J. C. Wheeler, and J. Wren 
This work was written as part of one of the author's official duties as an Employee of the United States Government and is therefore a work of the United States Government. In accordance with 17 U.S.C. 105, no copyright protection is available for such works under U.S. Law. Access to this work was provided by the University of Maryland, Baltimore County (UMBC) ScholarWorks@UMBC digital repository on the Maryland Shared Open Access (MD-SOAR) platform.

\section{Please provide feedback}

\section{Please support the ScholarWorks@UMBC repository by emailing scholarworks-group@umbc.edu and telling us what having access to this work means to you and why it's important to you. Thank you.}




\title{
OPTICAL LIGHT CURVE AND COOLING BREAK OF GRB 050502A
}

\author{
S. A. Yost ${ }^{1}$ K. Alatalo, ${ }^{1,2}$ E. S. Rykoff, ${ }^{1}$ F. Aharonian, ${ }^{3}$ C. W. Akerlof, ${ }^{1}$ M. C. B. Ashley, ${ }^{4}$ C. H. Blake, ${ }^{5}$ J. S. Bloom, ${ }^{2}$ \\ M. Boettcher, ${ }^{6}$ E. E. Falco, ${ }^{5}$ E. GöĞüș, ${ }^{7}$ T. Güver, ${ }^{8}$ J. P. Halpern, ${ }^{9}$ D. Horns, ${ }^{3}$ M. Joshi, ${ }^{6} \ddot{U}_{\text {. KiziloǦlu, }}{ }^{10}$ T. A. McKay, ${ }^{1}$ \\ N. Mirabal, ${ }^{1}$ M. Özel, ${ }^{11}$ A. Phillips, ${ }^{4}$ R. M. Quimby, ${ }^{12}$ W. Rujopakarn, ${ }^{1}$ B. E. Schaefer, ${ }^{13}$ J. C. Shields, $^{6}$ \\ M. Skrutskie, ${ }^{14}$ D. A. Smith, ${ }^{1}$ D. L. Starr, ${ }^{15}$ H. F. Swan, ${ }^{1}$ A. Szentgyorgyi, ${ }^{5}$ W. T. Vestrand, ${ }^{16}$ \\ J. C. WheELER, ${ }^{12}$ AND J. WREN ${ }^{16}$ \\ Received 2005 August 1; accepted 2005 September 16
}

\begin{abstract}
We present light curves of the afterglow of GRB $050502 \mathrm{~A}$, including very early data at $t-t_{\mathrm{GRB}}<60 \mathrm{~s}$. The light curve is composed of unfiltered ROTSE-IIIb optical observations from $44 \mathrm{~s}$ to $6 \mathrm{hr}$ postburst, $R$-band MDM observations from 1.6 to $8.4 \mathrm{hr}$ postburst, and PAIRITEL $J H K_{s}$ observations from 0.6 to $2.6 \mathrm{hr}$ postburst. The optical light curve is fit by a broken power law, where $t^{\alpha}$ steepens from $\alpha=-1.13 \pm 0.02$ to $-1.44 \pm 0.02$ at $\sim 5700 \mathrm{~s}$. This steepening is consistent with the evolution expected for the passage of the cooling frequency $\nu_{c}$ through the optical band. Even in our earliest observation at $44 \mathrm{~s}$ postburst, there is no evidence that the optical flux is brighter than a backward extrapolation of the later power law would suggest. The observed decay indices and spectral index are consistent with either an ISM or a wind fireball model, but slightly favor the ISM interpretation. The expected spectral index in the ISM interpretation is consistent within $1 \sigma$ with the observed spectral index $\beta=-0.8 \pm 0.1$; the wind interpretation would imply a spectral index slightly $(\sim 2 \sigma)$ shallower than observed. A small amount of dust extinction at the source redshift could steepen an intrinsic spectrum sufficiently to account for the observed value of $\beta$. In this picture, the early optical decay, with the peak at or below $4.7 \times 10^{14} \mathrm{~Hz}$ at $44 \mathrm{~s}$, requires very small electron and magnetic energy partitions from the fireball.
\end{abstract}

Subject heading: gamma rays: bursts

\section{INTRODUCTION}

Gamma-ray burst (GRB) afterglows are typically observed to decay as power laws in time (as reviewed by, e.g., Piran 2005). The leading afterglow model is the synchrotron fireball (Mészáros \& Rees 1997; Sari et al. 1998). It describes the afterglow as synchrotron emission from shock-accelerated electrons with a broken power-law spectral energy distribution, with several characteristic break frequencies. When the typical synchrotron frequency $\left(\nu_{m}\right)$ or the cooling frequency $\left(\nu_{c}\right)$ passes through the optical bands, the model predicts a break in the light curve.

The fireball model's spectral breaks have different power-law indices, depending on the circumburst medium (as discussed by Mészáros et al. 1998). The different resulting light curves allow important physical distinctions to be inferred from early afterglow observations. One example is the anticipated difference between a constant circumburst density (called interstellar me-

\footnotetext{
1 University of Michigan, 2477 Randall Laboratory, 450 Church Street, Ann Arbor, MI 48104; sayost@umich.edu, kalatalo@umich.edu, erykoff@umich.edu, akerlof@umich.edu, tamckay@umich.edu,wiphu@umich.edu,donaldas@umich .edu, hswan@umich.edu.

2 Astronomy Department, 601 Campbell Hall, University of California, Berkeley, CA 94720; kalatalo@berkeley.edu, jbloom@astron.berkeley.edu.

3 Max-Planck-Institut für Kernphysik, Saupfercheckweg 1, 69117 Heidelberg, Germany; felix.aharonian@mpi-hd.mpg.de, horns@mpi-hd.mpg.de.

4 School of Physics, Department of Astrophysics and Optics, University of New South Wales, Sydney, NSW 2052, Australia; mcba@phys.unsw.edu.au, a.phillips@unsw.edu.au.

5 Harvard College Observatory, Cambridge, MA 02138; cblake@cfa.harvard .edu, efalco@cfa.harvard.edu, aszentgyorgyi@cfa.harvard.edu.

6 Ohio University, Athens, OH 45701; boettchm@ohio.edu, manasvita.joshi.1@ ohio.edu,shields@phy.ohiou.edu.

7 Sabanci University, Orhanli-Tuzla 34956 Istanbul, Turkey; ersing@sabanciuniv .edu.

${ }^{8}$ Istanbul University Science Faculty, Department of Astronomy and Space Sciences, 34119, University-Istanbul, Turkey, tolga@istanbul.edu.tr.
}

dium [ISM]) and a $r^{-2}$ density gradient (called wind, as it resembles the environment produced by a steady mass-loss wind outflow). The GRB follow-up community has worked to produce ever-earlier observations in order to detect light-curve breaks.

The passage of the cooling frequency, $\nu_{c}$, has been inferred from changes in the broadband spectral index. The first such example was GRB 970508 (Galama et al. 1998), for which the optical-to-X-ray slope steepened as if $\nu_{c}$ were passing between them. Direct evidence of a cooling break passage in light curves is rarer. GRB 030329 showed a shallow change of slope at $t<$ 0.25 days, postulated to be the cooling break (Sato et al. 2003), although the standard fireball picture is complicated in that case, possibly with "layered" ejecta giving two jets and two jet breaks (Berger et al. 2003). More recently, Huang et al. (2005) found a shallow break in the GRB 040924 afterglow to be better explained by $\nu_{c}$ than by a jet break.

Here we report observations of the GRB 050502A afterglow in unfiltered optical and $R J H K_{s}$ bands. The optical data span nearly three logarithmic decades in time and begin at $t<1$ minute after the start of the GRB. We discuss the light curves in the context of the fireball model.

\footnotetext{
9 Columbia Astrophysics Lab, Columbia University, 550 West 120th Street, Mail Code 5230, New York, NY 10027-6601; jules@astro.columbia.edu.

${ }_{10}$ Middle East Technical University, 06531 Ankara, Turkey; umk@astroa physics.metu.edu.tr.

${ }_{11}$ Çanakkale Onsekiz Mart Üniversitesi, Terzioğlu 17020, Çanakkale, Turkey; me_ozel@ibu.edu.tr.

${ }^{12}$ Department of Astronomy, University of Texas, Austin, TX 78712; quimby@ astro.as.utexas.edu,wheel@astro.as.utexas.edu.

${ }^{13}$ Department of Physics and Astronomy, Louisiana State University, Baton Rouge, LA 70803; schaefer@1su.edu.

14 Department of Astronomy, University of Virginia, P.O. Box 3818, Charlottesville, VA 22903; mfs4n@virginia.edu.

${ }^{15}$ Gemini Observatory, Hilo, HI 96720; dan@pairitel.org.

${ }^{16}$ Los Alamos National Laboratory, NIS-2 MS D436, Los Alamos, NM 87545; vestrand@lanl.gov, jwren@nis.lanl.gov.
} 


\section{OBSERVATIONS}

This paper's optical and near-IR light curves are the result of three observation teams with different instruments at separate sites: the Robotic Optical Transient Search Experiment (ROTSE-III), the Peters Automated Infrared Imaging Telescope (PAIRITEL), and MDM.

The ROTSE-III array is a worldwide network of $0.45 \mathrm{~m}$ robotic, automated telescopes, built for fast $(\sim 6 \mathrm{~s})$ responses to GRB triggers from satellites such as the High Energy Transient Explorer $(H E T E-2)$ and Swift. They have a wide $(1.85 \times 1.85)$ field of view imaged onto a Marconi $2048 \times 2048$ back-illuminated thinned CCD and operate without filters, with a bandpass from approximately 400 to $900 \mathrm{~nm}$. ROTSE-IIIb is located at the McDonald Observatory in Texas. The ROTSE-III systems are described in detail in Akerlof et al. (2003).

The $1.3 \mathrm{~m}$ diameter PAIRITEL is a fully automated incarnation of the system used for the Two Micron All Sky Survey (2MASS). It is located at Mount Hopkins Ridge, Arizona. The camera consists of three $256 \times 256$ Near-Infrared Camera and Multi-Object Spectrometer (NICMOS3) arrays that simultaneously image the same $8.5 \times 8.5$ portion of the sky at $J, H$, and $K_{s}$ bands (central wavelengths $1.2,1.6$, and $2.2 \mu \mathrm{m}$ ).

The MDM Observatory is located at Kitt Peak, Arizona. It includes the $1.3 \mathrm{~m}$ McGraw-Hill telescope, which covers an $8.7 \times 8.7$ field of view. This instrument operates with a standard set of filters. The camera is a SITe $1024 \times 1024$ thinned, backside-illuminated CCD, with a pixel scale of 0."508.

On 2005 May 2, the International Gamma-Ray Astrophysics Laboratory (INTEGRAL) detected GRB 050502A (INTEGRAL trigger 2484) at 02:13:57 UT. The position was distributed as a GRB Coordinates Network (GCN) notice at 02:14:36 UT, with a $3^{\prime}$ radius error box, $39 \mathrm{~s}$ after the start of the burst (Gotz et al. 2005). The burst had a duration of $21 \mathrm{~s}$, with a fluence of $1.4 \times$ $10^{-6}$ ergs $\mathrm{cm}^{-2}$ in the $20-200 \mathrm{keV}$ band (Gotz \& Mereghetti 2005).

ROTSE-IIIb responded automatically to the GCN notice in $5.0 \mathrm{~s}$ with the first exposure starting at 02:14:41.0 UT, $44 \mathrm{~s}$ after the burst and only $23 \mathrm{~s}$ after the cessation of $\gamma$-ray activity. The automated scheduler began a program of ten $5 \mathrm{~s}$ exposures, ten $20 \mathrm{~s}$ exposures, and $41260 \mathrm{~s}$ exposures. The first exposure was taken during evening twilight hours, and ROTSE-IIIb was able to follow the burst position until the morning twilight. The early images were affected by scattered clouds and a bright sky background. Near-real-time analysis of the ROTSE-III images detected a 14th magnitude fading source at $\alpha=13^{\mathrm{h}} 29^{\mathrm{m}} 46^{\mathrm{s}} 3, \delta=+42^{\circ} 40^{\prime} 27^{\prime \prime} .7$ (J2000.0) that was not visible on the Digitized Sky Survey ${ }^{17}$ red plates, which we reported via the GCN Circular e-mail exploder within 40 minutes of the burst (Yost et al. 2005).

The PAIRITEL instrument on Mount Hopkins received the GRB 050502A trigger before dusk and generated a rapid response Target of Opportunity ( $\mathrm{ToO})$. When an interruption is generated by a new burst at nighttime, a new set of observations is queued, overriding all other targets. Typical time from GCN alert to slew is $10 \mathrm{~s}$, and typical slew times are 1-2 minutes. Since this burst occurred before nighttime science operations, it overrode the scheduled observations related to telescope pointing. This behavior yielded a bad pointing model in the first set of observations and has subsequently been prohibited. The first set of usable imaging began at 02:52:39.5 UT, or approximately 39 minutes after the trigger. Several imaging epochs were conducted over the following $5 \mathrm{hr}$.

17 See http://archive.stsci.edu/cgi-bin/dss_form.
PAIRITEL images were acquired with double-correlated sampling with effective exposure times of $7.848 \mathrm{~s}$ and dithered over several positions during a single epoch of observation. Typical total integration times are between 2 and 30 minutes with approximately $10-30$ different dither positions.

The MDM Observatory began $R$-band observations $1.8 \mathrm{hr}$ after the burst, following the initial ROTSE GCN report. Twentyone exposures were taken of the GRB field, spanning a total of $6.4 \mathrm{hr}$.

\section{DATA REDUCTIONS}

The three diverse data sets required different photometric reductions. All the data described below are given in Table 1.

The ROTSE-IIIb images were dark-subtracted and flat-fielded with its standard pipeline. The flat field was generated from 30 twilight images. SExtractor (Bertin \& Arnouts 1996) was applied for the initial object detection. The images were then processed with a customized version of the DAOPHOT point-spread function (PSF) fitting package (Stetson 1987) that has been ported to the IDL Astronomy User's Library (Landsman 1995). The PSF is calculated for each image from a set of $\sim 50$ well-measured stars within $12^{\prime}$ of the target location. The PSF is fit simultaneously to groups of stars using the nstar procedure (Stetson 1987). Relative photometry was then performed using 13 neighboring stars.

ROTSE-III magnitudes are calibrated as $R$-equivalent to accommodate ROTSE's peak optical bandpass sensitivity at red wavelengths. The $R$-equivalent magnitude zero point is calculated from the median offset to the USNO $1 \mathrm{~m} R$-band standard stars (Henden 2005) in the magnitude range $13.5<V<20.0$, with the color of typical stars in the field such that $0.4<V-$ $R<1.0$. As we have no data on afterglow color information at the early time, no additional color corrections have been applied to the unfiltered data, and the magnitudes quoted are then treated as $R$ band and referred to as $C_{R}$.

The PAIRITEL data were processed through its custom reductions pipeline and then combined into mosaics as a function of epoch and filter. The mosaicking used a cross-correlation between reduced images to find the pixel offsets for the dithers. Final images were constructed using a static bad-pixel mask and drizzling (Fruchter \& Hook 1997). Bias and sky frames were obtained for each frame by median-combining several images before and after that frame. Flat fields are applied from archival sky flats and are known to be highly stable over long periods of time. Aperture photometry was performed in a 3.5 pixel radius aperture (the average seeing FWHM was 2.4 pixels). The PAIRITEL photometric zero points for each of $J, H$, and $K_{s}$ were determined from 2MASS catalog stars, of which there are $\sim 20$ in the field.

The MDM data were processed using standard IRAF DAOPHOT procedures. Aperture photometry was performed in a 1."5 radius aperture (average seeing was $\sim 1$ 1.5) centered on the optical transient (OT) and nearby field stars. Instrumental magnitudes were then transformed to the $R$-system using the latest calibration provided by Henden (2005), using differential photometry for each image with respect to two Henden stars, the only ones available in the MDM field (R.A. $=13^{\mathrm{h}} 30^{\mathrm{m}} 04^{\mathrm{s}} 3$, decl. $=+42^{\circ} 41^{\prime} 06^{\prime \prime} 0$ and R.A. $=13^{\mathrm{h}} 29^{\mathrm{m}} 59^{\mathrm{s}} .8$, decl. $=+42^{\circ} 43^{\prime} 00^{\prime \prime} 2$, both J2000).

\section{RESULTS}

In order to analyze the results, we convert all magnitudes from Table 1 to spectral flux densities. For the $R$ (or ROTSE $C_{R}$ ) magnitudes, we use the effective frequencies and zero-point fluxes of Bessell (1979). The $J H K_{s}$ magnitudes are converted using 
TABLE 1

Optical Photometry for GRB 050502A

\begin{tabular}{|c|c|c|c|c|}
\hline Telescope & Filter & $\begin{array}{l}t_{\text {start }} \\
\text { (s) }\end{array}$ & $\begin{array}{l}t_{\text {end }} \\
\text { (s) }\end{array}$ & Magnitude \\
\hline \multirow[t]{23}{*}{ ROTSE-IIIb ........................ } & None & 44.0 & 49.0 & $14.28 \pm 0.11$ \\
\hline & None & 59.0 & 78.9 & $14.32 \pm 0.09$ \\
\hline & None & 88.5 & 108.0 & $14.71 \pm 0.16$ \\
\hline & None & 117.2 & 136.8 & $15.08 \pm 0.23$ \\
\hline & None & 146.7 & 180.5 & $15.63 \pm 0.23$ \\
\hline & None & 190.0 & 239.6 & $15.92 \pm 0.24$ \\
\hline & None & 248.7 & 327.7 & $16.19 \pm 0.33$ \\
\hline & None & 337.5 & 416.7 & $16.58 \pm 0.22$ \\
\hline & None & 426.5 & 546.1 & $17.04 \pm 0.20$ \\
\hline & None & 555.3 & 754.4 & $17.30 \pm 0.21$ \\
\hline & None & 763.6 & 962.7 & $17.50 \pm 0.28$ \\
\hline & None & 971.9 & 1309.9 & $17.75 \pm 0.24$ \\
\hline & None & 1319.7 & 1658.2 & $18.50 \pm 0.25$ \\
\hline & None & 1667.4 & 2214.4 & $18.66 \pm 0.13$ \\
\hline & None & 2224.1 & 2908.7 & $18.57 \pm 0.14$ \\
\hline & None & 2918.0 & 3881.3 & $19.09 \pm 0.11$ \\
\hline & None & 3890.5 & 5075.0 & $19.52 \pm 0.19$ \\
\hline & None & 5084.1 & 6741.0 & $19.84 \pm 0.22$ \\
\hline & None & 6750.1 & 8823.3 & $20.14 \pm 0.18$ \\
\hline & None & 8832.5 & 11670.7 & $20.90 \pm 0.29$ \\
\hline & None & 11680.6 & 15415.2 & $20.95 \pm 0.27$ \\
\hline & None & 15425.1 & 20274.6 & $21.34 \pm 0.33$ \\
\hline & None & 20284.1 & 28421.6 & $<21.67$ \\
\hline \multirow[t]{21}{*}{ 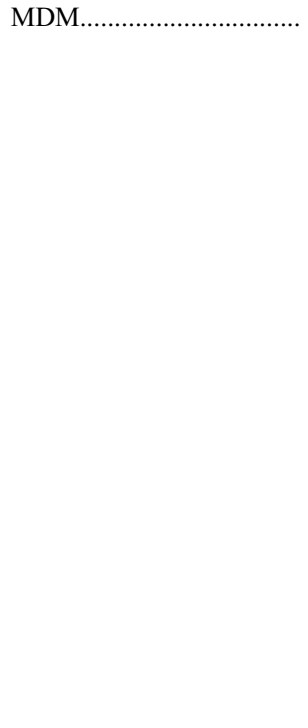 } & $R$ & 5816.0 & 6416.0 & $19.61 \pm 0.03$ \\
\hline & $R$ & 6458.0 & 7058.0 & $19.79 \pm 0.03$ \\
\hline & $R$ & 7105.0 & 7705.0 & $20.03 \pm 0.03$ \\
\hline & $R$ & 7750.0 & 8350.0 & $20.17 \pm 0.03$ \\
\hline & $R$ & 8386.0 & 8986.0 & $20.32 \pm 0.03$ \\
\hline & $R$ & 9025.0 & 9625.0 & $20.37 \pm 0.04$ \\
\hline & $R$ & 9928.0 & 10828.0 & $20.50 \pm 0.04$ \\
\hline & $R$ & 10871.0 & 11771.0 & $20.65 \pm 0.04$ \\
\hline & $R$ & 11812.0 & 12712.0 & $20.77 \pm 0.06$ \\
\hline & $R$ & 12750.0 & 13650.0 & $20.79 \pm 0.08$ \\
\hline & $R$ & 13687.0 & 14587.0 & $20.91 \pm 0.06$ \\
\hline & $R$ & 14621.0 & 15461.0 & $21.03 \pm 0.06$ \\
\hline & $R$ & 15645.0 & 16545.0 & $21.17 \pm 0.06$ \\
\hline & $R$ & 16587.0 & 17487.0 & $21.23 \pm 0.06$ \\
\hline & $R$ & 17537.0 & 18737.0 & $21.38 \pm 0.06$ \\
\hline & $R$ & 18797.0 & 19997.0 & $21.49 \pm 0.06$ \\
\hline & $R$ & 20115.0 & 21915.0 & $21.54 \pm 0.05$ \\
\hline & $R$ & 22041.0 & 23841.0 & $21.77 \pm 0.06$ \\
\hline & $R$ & 24057.0 & 25857.0 & $21.88 \pm 0.08$ \\
\hline & $R$ & 26358.0 & 28158.0 & $22.08 \pm 0.11$ \\
\hline & $R$ & 28319.0 & 30119.0 & $22.07 \pm 0.19$ \\
\hline \multirow{20}{*}{ PAIRITEL } & $J$ & 2322.5 & 3112.4 & $17.38 \pm 0.10$ \\
\hline & $J$ & 4942.0 & 6335.4 & $18.56 \pm 0.21$ \\
\hline & $J$ & 6365.4 & 7583.0 & $18.32 \pm 0.17$ \\
\hline & $J$ & 8158.9 & 9903.5 & $<19.25$ \\
\hline & $J$ & 9970.9 & 11722.3 & $<19.07$ \\
\hline & $J$ & 11790.4 & 13538.4 & $<19.21$ \\
\hline & $J$ & 13568.0 & 15316.8 & $<19.36$ \\
\hline & $J$ & 15382.5 & 17134.5 & $<19.40$ \\
\hline & $J$ & 17201.3 & 17979.7 & $<19.18$ \\
\hline & $J$ & 18951.5 & 20742.1 & $<19.40$ \\
\hline & $H$ & 2322.5 & 3112.4 & $16.64 \pm 0.10$ \\
\hline & $H$ & 4942.0 & 6335.4 & $17.70 \pm 0.23$ \\
\hline & $H$ & 6365.4 & 7583.0 & $17.77 \pm 0.23$ \\
\hline & $H$ & 8150.3 & 9903.5 & $<18.55$ \\
\hline & $H$ & 9970.9 & 11722.3 & $<18.19$ \\
\hline & $H$ & 11790.4 & 13538.4 & $<18.30$ \\
\hline & $H$ & 13568.0 & 15316.8 & $<18.32$ \\
\hline & $H$ & 15382.5 & 17134.5 & $<18.42$ \\
\hline & $H$ & 17201.3 & 17979.7 & $<18.10$ \\
\hline & $K_{s}$ & 2322.5 & 3112.4 & $15.85 \pm 0.11$ \\
\hline
\end{tabular}

TABLE 1-Continued

\begin{tabular}{ccrcc}
\hline \hline Telescope & Filter & \multicolumn{1}{c}{$\begin{array}{c}t_{\text {start }} \\
(\mathrm{s})\end{array}$} & \multicolumn{1}{c}{$\begin{array}{c}t_{\text {end }} \\
(\mathrm{s})\end{array}$} & Magnitude \\
\hline$K_{s}$ & 4942.0 & 6335.4 & $16.67 \pm 0.18$ \\
$K_{s}$ & 6365.4 & 7583.0 & $16.84 \pm 0.22$ \\
$K_{s}$ & 8150.3 & 9903.5 & $17.36 \pm 0.31$ \\
$K_{s}$ & 9970.9 & 11722.3 & $<17.33$ \\
$K_{s}$ & 11790.4 & 13538.4 & $<17.35$ \\
$K_{s}$ & 13568.0 & 15316.8 & $<17.57$ \\
$K_{s}$ & 15382.5 & 17134.5 & $<17.74$ \\
$K_{s}$ & 17201.3 & 17979.7 & $<17.21$ \\
$K_{s}$ & 18951.5 & 20742.1 & $<17.74$ \\
\hline
\end{tabular}

Note.-All times are in seconds since the burst time, 02:13:57 UT (see § 2).

Cohen et al. (2003). There is scant Galactic extinction at the high Galactic latitude of the transient. However, we do correct the fluxes for $0.028,0.01,0.006$, and 0.004 mag of extinction in the $R$ (and $\left.C_{R}\right), J, H$, and $K_{s}$ bands, respectively, as found from the extinction estimates of Schlegel et al. (1998).

Prochaska et al. (2005) established the source redshift, $z=$ 3.793. At such high $z$, absorption from the Ly $\alpha$ forest becomes important in the ROTSE-III bandpass. The bandpass covers the wavelength range of approximately $B-I$, but the Ly $\alpha$ absorption from this $z$ significantly depresses wavelengths corresponding to $B$ and $V$. Ordinarily, the blue spectrum of an OT would cause an overestimation of the $C_{R}$-band flux from ROTSE instrumental magnitudes, but in this case extinction across a significant fraction of the bandpass makes the conversion using the zero point from unextincted field stars underestimate the $C_{R}$-band flux. As a result, we expect a color term between the ROTSE $C_{R}$ and the MDM $R$ magnitudes. The precise color term is a complicated function of the $\operatorname{Ly} \alpha$ absorption and the OT spectrum. We fit a constant color term parameter when examining $R$ and $C_{R}$ data together as a constant multiplicative offset of the ROTSE points relative to the MDM data. This constancy presumes that any OT color changes within the ROTSE bandpass have a small effect on the color term parameter compared to the data uncertainties.

The light curves are plotted in Figure 1. The $R$-band light curve appears to be a broken power law. The need for a break is made obvious by the precise MDM observations at later times. The first (ROTSE) point in the light curve at $t-t_{\mathrm{GRB}}=44 \mathrm{~s}$ seems underluminous relative to subsequent decay. The IR data are less well sampled, but can be described as a set of power laws with decay rates similar to those seen in the $R$ band.

To quantify these trends, we fit the data using power-law and broken power-law forms. The smooth broken power-law form is

$$
f(t)=f_{0} 2^{1 / s}\left[\left(t / t_{b}\right)^{-s \alpha_{1}}+\left(t / t_{b}\right)^{-s \alpha_{2}}\right],
$$

where $t_{b}$ is the break time, $f_{0}$ is the flux at the break time, $\alpha_{1}$ and $\alpha_{2}$ are the two power-law indices, and $s$ is a sharpness parameter. The data (Fig. 1) clearly require a very sharp break transition. We use the form above for convenience, but do not fit the sharpness, setting $s$ at a very high value $(s=125)$. As noted above, we also fit a constant multiplicative factor for the ROTSE $C_{R}$ points, as a color term. The ROTSE points after the break observed in the data have significant uncertainties. We expect that any color change that may be associated with the break will not change the color term significantly in comparison to the ROTSE error bars.

The "separate" fit uses the form discussed above for the $R$-band data and individual power laws for each IR band (there is 


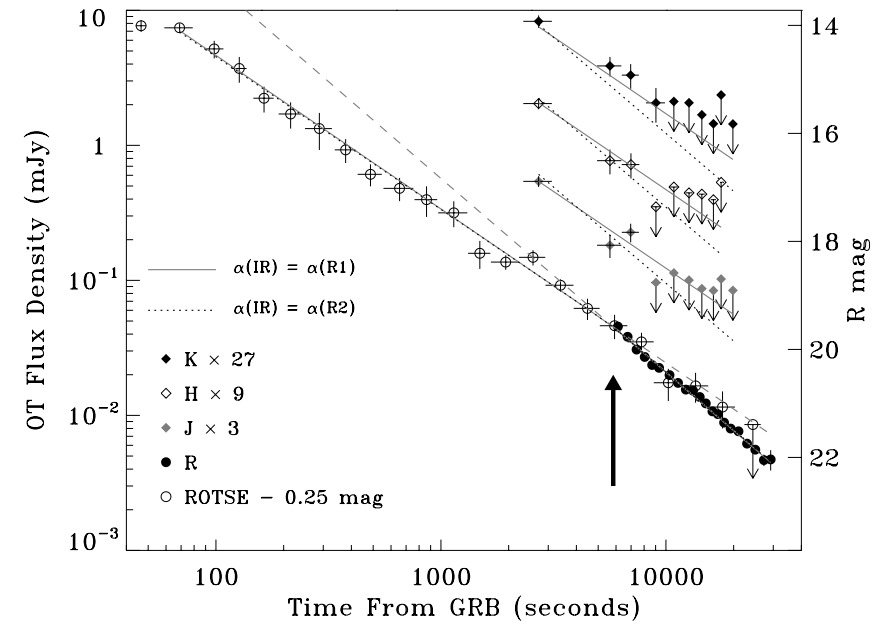

FIG. 1.- - Light curves of the optical-IR data presented in this paper. These are converted to fluxes as described in $\S 4$, and the $J, H$, and $K_{s}$ bands are offset by factors of 3,9, and 27, respectively, for clarity. The ROTSE points are unfiltered and calibrated to $R\left(C_{R}\right)$. These points are offset by a color term of $0.25 \mathrm{mag}$, a value determined from the $R / C_{R}$-band fit (see $\S 4$ ). Two fit models from Table 2 are overplotted. Each fits the bands simultaneously, scaling flux density $f_{\nu} \propto \nu^{\beta} t^{\alpha}$, with a break in $R$. The magnitude of the $R$ light-curve break matches the passage of the cooling frequency $\nu_{c}$. The solid fit requires $\alpha(\mathrm{IR})=\alpha_{R 1}$, the initial $R$-band decay, and the dotted fit requires $\alpha(\mathrm{IR})=\alpha_{R 2}$, the final $R$-band decay. The dashed lines show the extrapolations of the initial and final $R$ temporal power laws (solid fit), and the arrow denotes the break time. Both fits are reasonable; $\alpha(\mathrm{IR})=\alpha_{R 1}$ is expected for an ISM model, and $\alpha(\mathrm{IR})=\alpha_{R 2}$ is expected for a wind model. However, the $\alpha(\mathrm{IR})=\alpha_{R 2}$ fit underestimates the $K_{s}$ data.

no substantial evidence for breaks in the IR data). This allows each band to have different temporal decays and flux normalizations.

The "multiband" fit connects the wavelength bands via a spectral index and coupled temporal decays, as expected in the fireball model. This fit uses the above functional form for $R$-band data and simultaneously fits single power laws for the IR of the form

$$
f\left(\nu_{i}, t\right)=f_{0 i}\left(\nu_{i} / \nu_{R}\right)^{\beta}\left(t / t_{b}\right)^{\alpha_{\mathrm{IR}}},
$$

where $\nu_{i}$ designates the frequency of an IR band. We attempt two "multiband" forms, $\alpha_{\mathrm{IR}}=\alpha_{1}$ (the initial $R$-band decay index) and $\alpha_{\mathrm{IR}}=\alpha_{2}$ (the final $R$-band decay index).

\subsection{Early Underluminosity?}

First, we examined the $R$-band light curve (ROTSE and MDM). A single power law does not produce an acceptable fit. We fit all $R$-band data (including $C_{R}$ ) to the broken power law + color term function described above and repeated the exercise excluding the first point. Both produced formally acceptable fits, but excluding the first point improved the fit notably from $\chi^{2}=52.8$ for 39 degrees of freedom (dof) to $\chi^{2}=44.9$ for 38 dof.

The first observation, at $44 \mathrm{~s}$ post-GRB, appears to plateau, not joining the overall decay seen later. The flux density is approximately $4 \sigma$ below the back extrapolation of the fit excluding the first point: marginal evidence for a deviation at early times from the later decay. While a single point cannot definitively establish such a deviation, the first point does not fit the overall later decay, and thus we exclude it from the light-curve fits discussed below and recorded in Table 2. The potential constraints placed by this first point are discussed in $\S 5.2$.

Overluminosity, or flux above the expectations from the subsequent decay, would be expected if a reverse shock's emission were observable as it passes through the ejecta at early times
TABLE 2

Fit Parameters AND $\chi^{2}$

\begin{tabular}{|c|c|c|}
\hline Parameter & Description & Fit Value \\
\hline \multicolumn{3}{|c|}{ Fits to the Individual Bands } \\
\hline$\alpha_{R 1} \ldots$ & Early $R$ power-law index & $-1.131 \pm 0.023$ \\
\hline$\alpha_{R 2} \ldots \ldots \ldots \ldots \ldots \ldots \ldots \ldots \ldots$ & Late $R$ power-law index & $-1.437 \pm 0.022$ \\
\hline$t_{b}(\mathrm{~s}) \ldots \ldots \ldots \ldots \ldots \ldots \ldots \ldots \ldots \ldots \ldots \ldots \ldots \ldots \ldots \ldots \ldots$ & $R$ break time & $5690 \pm 750$ \\
\hline$f_{0, R}\left(t_{b}\right)(\mu \mathrm{Jy}) \ldots \ldots \ldots \ldots$ & $f_{\nu}(R)$ at $t_{b}$ & $46.7 \pm 8.7$ \\
\hline$\kappa(\mathrm{mag})$ & ROTSE color term & $-0.250 \pm 0.073$ \\
\hline$\chi_{R}^{2} \ldots \ldots \ldots \ldots \ldots \ldots \ldots \ldots \ldots \ldots \ldots \ldots \ldots \ldots \ldots \ldots$ & $\chi^{2}$ of $R \mathrm{fit} / \mathrm{dof}$ & $44.9 / 38$ \\
\hline$\alpha_{J} \ldots \ldots \ldots \ldots \ldots \ldots \ldots$ & $J$ power-law index & $-1.33 \pm 0.18$ \\
\hline$F_{J}\left(t_{0}\right)(\mu \mathrm{Jy}) \ldots \ldots \ldots \ldots \ldots$ & $f_{\nu}(J)$ of 1st IR epoch & $182 \pm 16$ \\
\hline$\chi_{J}^{2} \ldots \ldots \ldots \ldots \ldots \ldots \ldots \ldots \ldots \ldots \ldots \ldots \ldots \ldots \ldots \ldots \ldots$ & $\chi^{2}$ of $J$ fit $/$ dof & $8.0 / 8$ \\
\hline$\alpha_{H} \ldots \ldots \ldots \ldots \ldots \ldots \ldots \ldots \ldots \ldots \ldots \ldots \ldots \ldots \ldots$ & $H$ power-law index & $-1.36 \pm 0.21$ \\
\hline$F_{H}\left(\mathrm{t}_{0}\right)(\mu \mathrm{Jy}) \ldots \ldots \ldots \ldots$ & $f_{\nu}(H)$ of 1 st IR epoch & $229 \pm 21$ \\
\hline$\chi_{H}^{2} \ldots \ldots \ldots \ldots \ldots \ldots \ldots \ldots \ldots$ & $\chi^{2}$ of $H$ fit $/$ dof & $3.6 / 7$ \\
\hline$\alpha_{K_{s}} \ldots \ldots \ldots \ldots \ldots \ldots \ldots \ldots \ldots$ & $K_{s}$ power-law index & $-1.17 \pm 0.17$ \\
\hline$F_{K_{s}}\left(\mathrm{t}_{0}\right)(\mu \mathrm{Jy}) \ldots \ldots \ldots \ldots$ & $f_{\nu}\left(K_{s}\right)$ of 1 st IR epoch & $316 \pm 32$ \\
\hline 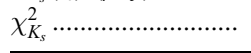 & $\chi^{2}$ of $K_{s}$ fit $/$ dof & $3.4 / 8$ \\
\hline \multicolumn{3}{|c|}{ Multiband Fit: NIR Decay Index $=\alpha_{R 1}$} \\
\hline$\alpha$ & Early power-law index & $-1.137 \pm 0.022$ \\
\hline$\alpha_{R 2} \ldots \ldots \ldots$ & Late power-law index ( $R$ only) & $-1.435 \pm 0.022$ \\
\hline$t_{b}(\mathrm{~s}) \ldots \ldots \ldots \ldots \ldots \ldots \ldots \ldots \ldots \ldots \ldots \ldots$ & $R$ break time & $5830 \pm 250$ \\
\hline$f_{0, R}\left(t_{b}\right)(\mu \mathrm{Jy}) \ldots \ldots \ldots \ldots$ & $f_{\nu}(R)$ at $t_{b}$ & $45.2 \pm 2.8$ \\
\hline & Spectral index $\nu^{\beta}$ & $-0.785 \pm 0.052$ \\
\hline$\kappa(\mathrm{mag})$ & ROTSE color term & $-0.263 \pm 0.066$ \\
\hline$\chi^{2} \ldots \ldots \ldots \ldots \ldots \ldots \ldots \ldots \ldots \ldots \ldots \ldots \ldots \ldots \ldots$ & $\chi^{2}$ of multiband fit/dof & $65.7 / 66$ \\
\hline \multicolumn{3}{|c|}{ Multiband Fit: NIR Decay Index $=\alpha_{R 2}$} \\
\hline$\alpha_{R}$ & Early & $-1.131 \pm 0.024$ \\
\hline ........ & Late power-law index & $-1.425 \pm 0.022$ \\
\hline$t_{b}(\mathrm{~s}) \ldots \ldots \ldots \ldots \ldots \ldots \ldots \ldots \ldots$ & $R$ break time & $5600 \pm 1800$ \\
\hline$f_{0, R}\left(t_{b}\right)(\mu \mathrm{Jy}) \ldots \ldots \ldots \ldots$ & $f_{\nu}(R)$ at $t_{b}$ & $47 \pm 21$ \\
\hline 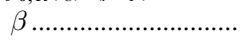 & Spectral index $\nu^{\beta}$ & $-0.641 \pm 0.055$ \\
\hline$\kappa(\mathrm{mag})$ & ROTSE color term & $-0.252 \pm 0.096$ \\
\hline$\chi^{2} / \operatorname{dof} \ldots \ldots \ldots \ldots \ldots \ldots \ldots \ldots \ldots \ldots$ & $\chi^{2}$ of multiband fit/dof & $68.2 / 66$ \\
\hline
\end{tabular}

comparable to our first observation (e.g., Mészáros \& Rees 1997; Sari \& Piran 1999). This is certainly not seen in our light curve. The maximum flux we observe in the first observation $(99 \%$ confidence upper limit) is only $90 \%$ of the back-extrapolated flux from the fit excluding the first data point. A flux level significantly above this extrapolation would have been readily measurable.

\subsection{Fitting the Frequency Bands Separately}

The $R$ (and $C_{R}$ ) band, excluding the first point, was fitted to the broken power law as described above, and each of the $J, H$, and $K_{s}$ bands was independently fitted to a power law. Nondetections are included as flux densities of $0 \pm f\left(m_{\lim }\right)$ to force the fit not to overestimate undetected fluxes. However, the elimination of nondetections from the fits does not change the parameters significantly. The resulting fit parameters are given in Table 2 . Overall, the total $\chi^{2}=60$ for 61 dof.

Due to the $\operatorname{Ly} \alpha$ absorption discussed above, the $R$-band fit requires a significant color term, $0.25 \mathrm{mag}$, for the ROTSE $C_{R}$ data relative to the filtered MDM $R$-measurements. With this systematic correction, the $R$-band light curve has a sharp break at a time $5700 \pm 800 \mathrm{~s}$ postburst. The $R$-band light curve break is from a decay index $\alpha$ of $-1.13 \pm 0.02$ to $-1.44 \pm 0.02$, a $\Delta \alpha=$ $-0.31 \pm 0.03$. In the fireball model, the expected steepening from a "jet break" (observing a lack of flux due to the edge of conical 
ejecta) is at least $\Delta \alpha=-0.75$ (Panaitescu \& Mészáros 1999; Sari et al. 1999). The only shallow steepening expected in the fireball model is from the passage of the cooling frequency. The model predicts $\Delta \alpha\left(\nu_{c}=\nu\right)=-0.25$, consistent with the observed break at the $1.8 \sigma$ level.

The NIR data are consistent with power-law fits. The fitted $J$, $H$, and $K_{s}$ decay rates lie between the initial and final $R$-band decay rates. They are internally consistent at the $1 \sigma$ level and thus show no evidence for NIR color changes. The NIR decay indices are not as tightly constrained as those describing the $R$ band, with individual uncertainties $\delta \alpha \approx 0.2$. As the $R$-band break is shallow, each NIR band's decay is consistent with either the initial or the final $R$-band decay index (at the $1.6 \sigma$ level or better).

Consistency with the initial $R$-band decay would be expected for an ISM fireball in which $\nu_{c}$ drops as $\propto t^{-1 / 2}$, while consistency with the final $R$-band decay would be expected for a wind fireball where $\nu_{c}$ rises as $\propto t^{1 / 2}$ (as reviewed by Piran 2005). The $K_{s}$ band is slightly more consistent with the ISM picture than with the wind; the $H$ and $J$ bands are slightly more consistent with the wind than with the ISM.

As evident from the poorly determined NIR decay rates, the NIR data do not have the sensitivity to determine the presence or absence of a light-curve break as shallow as that observed in the $R$ band. Moreover, the NIR time coverage is from 2320 to $20,700 \mathrm{~s}$, and the observed $R$-band break is at $5700 \pm 800 \mathrm{~s}$. Even if the NIR data were more sensitive given the timing of the $R$-band lightcurve break, in the ISM (wind) model prediction, $\nu_{c}$ would not be observed falling (rising) to (from) the $J$ band during the NIR coverage. The ISM model predicts that $\nu_{c}$ would fall from $R$ to $J$ only at $t \sim 21,000 \pm 3000 \mathrm{~s}$. The wind model predicts that $\nu_{c}$ would rise from $J$ to $R$, with the break in $J$ at $t \sim 1600 \pm 200 \mathrm{~s}$.

\subsection{Multiband Fitting}

Given no obvious color changes, we also fitted the multiband function as described in $\S \S 4.1$ and 4.2. We gave initial parameters to search for two cases, with the NIR decay being the power-law behavior either of the initial $R$-band light curve decay or of the final $R$-band decay. The two results are shown in Table 2 . The $R$-decay indices, the $R$-band break time, and the $R$-flux at the break time do not change significantly. The break amplitude $\Delta \alpha$ is consistent with the cooling break passage at the $1.5 \sigma$ level in both cases.

Both multiband fits are formally quite good, with $\chi^{2} /$ dof $\approx 1$. The data are insufficiently constraining to distinguish between $\alpha_{\mathrm{IR}}=\alpha_{R 1}$ (ISM expectation) and $\alpha_{\mathrm{IR}}=\alpha_{R 2}$ (wind expectation). We show both of these fits in Figure 1, where the shallower $\beta$ favored by the $\alpha_{\mathrm{IR}}=\alpha_{R 2}$ model and the steeper IR decay make the fitted model underestimate all the $K_{s}$ data slightly. As the fit is nevertheless good, this only mildly favors $\alpha_{\mathrm{IR}}=\alpha_{R 1}$ and the ISM interpretation.

\subsection{Spectral Index $\beta$}

The optical-NIR spectrum of the data is well fit by a power law, $f_{\nu} \propto \nu^{\beta}$. This is observed in the NIR data alone $\left(J H K_{s}\right.$ at one common epoch), as well as $R J H K_{s}$ data (interpolating the $R$-band point). There is no significant change across observation epochs, as there is no evidence for color changes from the light curves. The temporal decays of the IR bands are not strongly constrained $(\delta \alpha \approx 0.2$, Table 2$)$, are all mutually consistent, and are all consistent (at $<2 \sigma$ ) with either the initial or the final $R$-band decay. Moreover, the complete data set is well fit by the "multiband" function that includes the spectral index $\beta$ to connect the bands.

The $J H K_{s}$ spectrum at the initial epoch yields $\beta=-0.9 \pm 0.3$, while $R J H K_{s}$ gives $\beta=-0.9 \pm 0.2$. These are not as well constrained as the $\beta$-fit using the multiband function. When $\alpha_{\mathrm{IR}}=\alpha_{R 1}, \beta=-0.79 \pm 0.05$ is fit, and when $\alpha_{\mathrm{IR}}=\alpha_{R 2}$, $\beta=-0.64 \pm 0.06$.

These results are all mutually consistent. Given their variation, we take our measure of the optical-NIR spectral index to be $\beta=$ $-0.8 \pm 0.1$. The observed $R$-band light-curve break produces color changes relative to the single power laws of the NIR bands, but they are not detectable, as we do not have good IR detections in the period from the break to the end of our NIR observations.

\section{DISCUSSION}

The power-law break in the $R$-band light curve is consistent with $\Delta \alpha=0.25$, the value expected for the passage of a cooling break, $\nu_{c}$, in a fireball model with either an ISM or wind circumburst density. The fireball model can also steepen a decay via a jet break, or via the passage of the typical frequency $\nu_{m}$ in the fastcooling $\left(\nu_{c}<\nu_{m}\right)$ case. The latter, for an observing frequency $\nu$, is the transition from $\nu_{c}<\nu<\nu_{m}$ to $\nu_{c}<\nu_{m}<\nu$. As previously noted, a jet break should be significantly steeper than the $\Delta \alpha \approx 0.3$ observed (Panaitescu \& Mészáros 1999; Sari et al. 1999). The $\nu_{m}$ passage in a fast-cooling case requires the initial decay to be $t^{-1 / 4}$ before the break (Piran 2005; see their $\S$ VIIB), which is not compatible with any of the light curves.

From the temporal indices, we conclude that we are observing the passage of $\nu_{c}$ through $R$ at $t \approx 6000 \mathrm{~s}$. In the ISM case, this is a passage from $\nu_{m}<\nu(R)<\nu_{c}$ to $\nu_{m}<\nu_{c}<\nu(R)$. In the wind case, it is from $\nu_{m}<\nu_{c}<\nu(R)$ to $\nu_{m}<\nu(R)<\nu_{c}$. Both require that $\nu_{m}$ is below the optical by the first fit data point at $t-t_{\mathrm{GRB}}=59 \mathrm{~s}$.

\subsection{Interpreting $\alpha-\beta$}

The fireball model predicts relations between observed temporal and spectral indices and the value $p\left[N(\gamma) \propto \gamma^{p}\right]$ of the power-law index for the spectral energy distribution of synchrotron-emitting electrons. The relations are functions of synchrotron spectral break ordering, the spectral segment, and whether the fireball is the ISM or wind case (compiled by Piran 2005). With an initial and a final decay index, there are two relations to determine $p$ for each assumed case (ISM or wind). Using the $R$-band decay indices from the "separate" fit gives values of $p: 2.51 \pm 0.03$ (from the initial $\alpha_{R 1}$ ) and $2.58 \pm 0.03$ (final $\alpha_{R 2}$ ) for the constant-density ISM case, and $2.17 \pm 0.03\left(\alpha_{R 1}\right)$ and $2.25 \pm 0.03\left(\alpha_{R 2}\right)$ assuming the wind case.

Given the relations between $p$ and $\alpha$, as well as those between $p$ and $\beta, \alpha$ and $\beta$ are related (again compiled in Piran 2005; see their $\S$ VII). With the $R$ light-curve break modeled by the passage of $\nu_{c}$ in either an ISM or a windlike medium, there are four possible $\alpha-\beta$ "closure" relations of $g(\alpha, \beta)=0$. We summarize them in Table 3 along with the values for the electron energy spectral index $p(\alpha)$ in these cases, and $p(\beta)$.

As before, the ISM model better represents the data. The ISM closure $g(\alpha, \beta)$ results are more consistent with 0 , but the wind cases do not deviate by more than $\approx 2 \sigma$. Similarly, the values of $p$ inferred from the ISM relations, $p(\alpha)$, better agree with its $p(\beta)$ than in the wind case, with a level of consistency similar to the closure relations.

The observed spectral index of $-0.8 \pm 0.1$ is consistent with either the ISM or wind picture, as seen in the closure relations, but is slightly steeper than the wind model expects (Table 3 ), by $\Delta \beta \approx 0.2$. At the source redshift of $z=3.793$ (Prochaska et al. 2005 ), the $K_{s}$ band in the observer frame is roughly $B$ band in the local frame, and the $R$ band is near-UV at $2 \times 10^{15} \mathrm{~Hz}$. Over this frequency range in the source frame, a dust extinction law such as that of the Large Magellanic Cloud (LMC) will be linear, leading to a simple power-law steepening of the spectrum (Fitzpatrick 
TABLE 3

Closure Relations for ( $R$-band) Decay Index $\alpha$, Spectral Index $\beta$

\begin{tabular}{|c|c|c|c|c|c|}
\hline Model & Relation $(=0)^{\mathrm{a}}$ & Result & $p(\alpha)$ & $p(\beta)$ & Expected $\beta[p(\alpha)]$ \\
\hline \multirow[t]{2}{*}{ ISM, initially $\nu_{\mathrm{m}}<\nu(R)<\nu_{c} \ldots \ldots \ldots$} & $\alpha_{1}-1.5 \beta$ & $0.07 \pm 0.15$ & $2.51 \pm 0.03$ & $2.6 \pm 0.2$ & $-0.76 \pm 0.02$ \\
\hline & $\left(\alpha_{2}-1.5 \beta\right)+0.25$ & $0.01 \pm 0.15$ & $2.58 \pm 0.03$ & $2.6 \pm 0.2^{\mathrm{b}}$ & $-0.79 \pm 0.02$ \\
\hline \multirow[t]{2}{*}{ Wind, initially $\nu_{m}<\nu_{c}<\nu(R) \ldots \ldots \ldots$} & $\left(\alpha_{1}-1.5 \beta\right)+0.25$ & $0.32 \pm 0.15$ & $2.17 \pm 0.03$ & $2.6 \pm 0.2^{\mathrm{c}}$ & $-0.59 \pm 0.02$ \\
\hline & $\left(\alpha_{2}-1.5 \beta\right)+0.5$ & $0.26 \pm 0.15$ & $2.25 \pm 0.03$ & $2.6 \pm 0.2$ & $-0.63 \pm 0.02$ \\
\hline
\end{tabular}

Notes.-The value of $\alpha_{1}$ is $\alpha_{R 1}$, and $\alpha_{2}$ is $\alpha_{R 2}$ from the Table 2 fit to the individual bands; $\beta$ is $-0.8 \pm 0.1$, as determined in $\S 4.4$. The multiband fits also satisfy the closure relations given here.

a As compiled in the review by Piran (2005).

b The value of $p$ is determined from $\beta$ assuming that prebreak the optical and NIR have a spectrum $\propto \nu^{-(p-1) / 2}$, and is the same postbreak.

${ }^{c}$ The value of $p$ is determined from $\beta$ assuming that postbreak the optical and NIR have a spectrum $\propto \nu^{-(p-1) / 2}$, and is the same prebreak.

\& Massa 1988; Reichart 2001). Dust extinction such as that observed in the Milky Way would have a significant bump in the middle of that frequency range, so we did not consider it. We use the LMC prescription of Reichart (2001) and determine that a small local extinction, $A(V) \approx 0.1$, would give $\Delta \beta=0.2$. The amount of extinction would be sufficient to provide an intrinsic spectrum compatible with the wind model. The extinction law prescribed for the Small Magellanic Cloud (SMC) bar is steeper than the LMC case and so, if applicable, should require less dust.

There cannot be a very large amount of extinction at the burst redshift. To get the observed spectrum with as little extinction as $A(V)=0.5$ requires a flat or rising intrinsic spectrum. Such an intrinsic spectrum is inconsistent with the fireball model.

Hurkett et al. (2005) presented the Swift X-Ray Telescope's (XRT) X-ray upper limits from observations later than those presented here. We compare the X-ray limits to the optical by extrapolating the $R$-band flux density to their epoch, using our fitted models. We convert their flux limit to a spectral flux density limit using the spectral information given, both for their earliest epoch at $\sim 50 \mathrm{ks}$ and for the overall observation. We find that the early optical spectral index of $\beta=-0.8$ is marginally consistent with the X-ray limit, overestimating the X-ray $90 \%$ upper limit by approximately a factor of 3 . (Given that the uncertainty of $\beta$ is 0.1 , note that $\beta=-0.9$ would only overestimate the $\mathrm{X}$-ray limit by a factor of 1.5 , and $\beta=-0.95$ would match it.) The expected initial intrinsic $\beta=-0.8$ (ISM model; Table 3) would be marginally consistent, but the expected $\beta=-0.6$ (wind; Table 3 ) would be inconsistent, overestimating the X-ray limit by at least a factor of 14 .

This X-ray-optical spectrum is consistent with the passage of $\nu_{c}$. With an ISM model, $\nu_{c}$ has passed below the optical, steepening the spectrum between $R$ and the X-ray at later times. With a wind profile, $\nu_{c}$ increases with time, placing it between $R$ and the X-ray at the time of the X-ray limit. However, $\nu_{c}$ would not get far above the optical, and the spectrum steepens between $\nu_{c}$ and the X-ray. In both cases, the steepening $\Delta \beta=-0.5$ above $\nu_{c}$ results in a model-predicted X-ray flux well below the Swift XRT limit.

\subsection{Fireball Model Constraints}

If the subluminosity of the earliest $R$-band observation (44 $\mathrm{s}$ ) were known to be due to the passage of the peak $\nu_{m}$, there would be three numerical constraints for the fireball model's spectral parameters: the peak time in $R$ band, the peak flux density value at that time, and the cooling break time at the $R$-band frequency. The early light-curve behavior is neither strong evidence for a rollover from an earlier flatter light-curve evolution, nor strong evidence for a particular physical mechanism for a rollover. There- fore, the first two constraints are inequalities; the peak $\nu_{m}$ could have passed the optical band earlier.

The observed decay from 44 to $6000 \mathrm{~s}$ requires that the passage $\nu_{m}=\nu(R)$ is occurring or has occurred at $t \leq 44 \mathrm{~s}$. If $\nu_{m}$ passed the $R$ band before $44 \mathrm{~s}$, the synchrotron spectral peak would be at a lower frequency and a higher flux density than that of the $R$ band. Thus, the peak flux density $f\left(\nu_{m}\right)$ at $44 \mathrm{~s}$ must be at least as bright as the initial $R$-band point.

The peak passage $\nu_{m}$ is quite early, having occurred at a time no later than 2.1 times the GRB duration measured by Gotz \& Mereghetti (2005). The cooling-break passage time of $\approx 5700 \mathrm{~s}$ is significant, placing $\nu_{c}$ in the optical at a fairly early time. This is quite early in comparison to several other cases where broadband data have indicated $\nu_{c}$ is above the optical at a time $\sim$ days postburst, e.g., GRB 970508 (Galama et al. 1998), GRB 000301C (Berger et al. 2001), or GRB 011211 (Jakobsson et al. 2003). The implication in the ISM case with $\nu_{c} \propto t^{-1 / 2}$ would be a low value of $\nu_{c}$ during the first observation, with $\nu_{c} \sim 5 \times 10^{15} \mathrm{~Hz}$ at $44 \mathrm{~s}$.

We consider the observational constraints in light of both the ISM and the wind models for the data. The synchrotron spectrum of a spherical fireball expanding into the ISM at a known redshift depends on five parameters: energy, $E$; density, $n$; the power-law slope of the electron energy distribution, $p$; the energy fraction partitioned to the electrons, $\epsilon_{e}$; and the energy partition to the magnetic fields, $\epsilon_{B}$ (see Piran 2005 and references therein). With three constraints and the value of $p$, we can put three of $E, n, \epsilon_{B}$, and $\epsilon_{e}$ in terms of the fourth, thus placing a limit on a combination of two parameters.

We use the equations of Granot \& Sari (2002) for the ISM slow-cooling case for $\nu_{m}, \nu_{c}$, and $F_{\nu_{m}}$. Adopting $p=2.55$, we find the numerical relations using the redshift $z=3.793$ (Prochaska et al. 2005) and a cosmology with $\Omega_{m}=0.3, \Omega_{\Lambda}=0.7, H_{0}=$ $65 \mathrm{~km} \mathrm{~s}^{-1} \mathrm{Mpc}^{-1}$ to determine the appropriate luminosity distance. We can then isolate

$$
\left(\epsilon_{e} / 0.1\right)\left(\epsilon_{B} / 0.01\right)^{1 / 3} \leq 0.024
$$

where $\epsilon_{e}$ and $\epsilon_{B}$ are scaled to $10 \%$ and $1 \%$, respectively, as these are reasonable expectations for the parameters from broadband fits in many afterglow cases (see, e.g., Panaitescu \& Kumar 2002; Yost et al. 2003). Within the simple fireball model that well describes this afterglow case in the optical-IR, the implication is that the microphysical energy partitions must be quite small. For example, if $\epsilon_{e}=1 \%$, then $\epsilon_{B}=0.01 \%$. Other parameters can be put in terms of these; thus, density $n \propto \epsilon_{B}^{-10 / 6}$. Keeping $n<$ $100 \mathrm{~cm}^{-3}$ requires $\epsilon_{B}$ to be not much less than $1 \%$.

We note that the microphysical parameter combination in equation (3) is proportional to the peak flux density at $44 \mathrm{~s}$ as 
$F_{\nu_{m}}^{-1 / 3}$, and proportional to the frequency of the peak $\nu_{m}$ at $44 \mathrm{~s}$ as $\nu^{1 / 2}$. Thus, if the peak was significantly below the $R$ band at that time, this combination of microphysical parameters would be notably smaller.

For a wind model, the physical parameter set is the same except that the density is parameterized by $A_{*}$, where $\rho=5 \times$ $10^{11} A_{*} r^{-2} \mathrm{~g} \mathrm{~cm}^{-1}$. We do a similar analysis using Granot \& Sari's (2002) equations. In this case, we find

$$
\left(\epsilon_{e} / 0.1\right)\left(\epsilon_{B} / 0.01\right)^{1 / 3} \leq 0.08 .
$$

Once again, the microphysical parameters must be small (and increasingly small as the initial value of $\nu_{m}$ is placed further below the $R$-band frequency).

There is an additional constraint:

$$
A_{*} \geq 0.0004\left(\epsilon_{e} / 0.1\right)^{2} .
$$

This inequality indicates that if $\nu_{m}<\nu(R)$ at the initial observation, $A_{*}\left(\epsilon_{e} / 0.1\right)^{-2}$ would be larger than 0.0004 . However, from the previous relation the low $\nu_{m}$ would require smaller microphysical parameters, $\epsilon_{e}$ or $\epsilon_{B}$, or both. If $\epsilon_{e}$ is decreased in the case where $\nu_{m}<\nu(R)$, then $A_{*}$ would remain low. A value $A_{*}=0.0004$ is a very small wind outflow parameter; $A_{*} \sim 1$ is observed in Wolf-Rayet stars (as reviewed by Willis 1991). The physical parameter constraints are somewhat easier to fulfill with the ISM fireball model than with the wind model.

\section{CONCLUSIONS}

We observe a shallow break of $\Delta \alpha \approx 0.3$ in the $R$ light curve of the GRB $050502 \mathrm{~A}$ afterglow at approximately $6000 \mathrm{~s}$ postburst. We note that $J, H$, and $K_{s}$ observations during this period, not as well sampled, do not constrain the presence or absence of such a light-curve break in the NIR. With the shallow $\Delta \alpha$, we conclude that the optical break represents the passage of the synchrotron cooling frequency $\nu_{c}$ through $R$.

The observed spectral index is $\beta=-0.8 \pm 0.1$. This is consistent with an ISM model for the broken $R$-band light curve, and slightly steeper than that expected in a wind model. The temporal and spectral index closure relations slightly favor an ISM over a wind interpretation. A small amount of dust at the host redshift would steepen an intrinsically flatter spectrum sufficiently to accommodate the wind interpretation.

There is no evidence for an overluminosity at our earliest observation, $44 \mathrm{~s}$ after the initial gamma rays. The first lightcurve point appears suppressed, but from a single point we cannot conclude that this is the case. At a minimum, the early decay requires $\nu_{m}$ at or below $\nu(R)$ at that time. Using that constraint and the observation of the passage of $\nu_{c}$ indicates that in both the ISM and the wind explanations, small microphysical energy partitions are required. The wind interpretation expects an exceptionally low wind outflow parameter $A_{*}$, which may again somewhat favor an ISM interpretation.
Guidorzi et al. (2005) found multicolor evidence for a lightcurve bump at $t \approx 1700-3400 \mathrm{~s}$. A single ROTSE data point during this period has a slight rise above the overall decay, but not at a significant level given its signal-to-noise ratio. $J H K_{S}$ sampling is insufficient to address whether the bump occurs at these wavelengths. Without the well-sampled MDM $R$-band observations presented here from 5800 to 28,000 s, Guidorzi et al. (2005) did not detect the passage of $\nu_{c}$ through $R$.

Guidorzi et al. (2005) found that the data favor a density variation over a refreshed shock as the source of the bump. In that case, the significance of the bump is greater than expected for $\nu(R)>\nu_{c}$ (see Nakar \& Piran 2003). For the wind case the cooling-break passage observed in the data set presented here would be from $\nu(R)>\nu_{c}$ to $\nu(R)<\nu_{c}$ at $\approx 6000 \mathrm{~s}$, so this again favors an ISM over a wind interpretation.

We note that the observed steeper, steady decay following the light-curve break is evident in the data over at least 0.7 logarithmic decades in time. Despite the evidence for a density variation in the data of Guidorzi et al. (2005), such an impulsive event cannot explain the sustained change in light-curve evolution seen here.

There are several lines of evidence, including the spectral index, the closure relations of spectral and temporal indices, and the optical bump seen by Guidorzi et al. (2005), which all somewhat favor an ISM model over a wind model for this afterglow. With Swift and INTEGRAL providing rapid triggers, and rapid response instruments such as ROTSE and PAIRITEL providing follow-up, the GRB community is accumulating bursts with prompt, detailed observations. Such data sets should further probe the physics of GRB afterglows.

ROTSE-III has been supported by NASA grant NNG04WC41G, NSF grant AST 04-07061, the Australian Research Council, the University of New South Wales, the University of Texas, and the University of Michigan. Work performed at LANL is supported through internal LDRD funding. Special thanks to the observatory staff at McDonald Observatory, especially David Doss. The MDM work has been supported by the National Science Foundation under grant 0206051 to J. P. H. The Peters Automated Infrared Imaging Telescope (PAIRITEL) is operated by the Smithsonian Astrophysical Observatory (SAO) and was made possible by a grant from the Harvard University Milton Fund, the camera loan from the University of Virginia, and the continued support of the SAO and University of California, Berkeley. Partial support is also supplied by a NASA Swift Cycle 1 Guest Investigator grant. The Digitized Sky Surveys were produced at the Space Telescope Science Institute under US Government grant NAG W-2166. The images of these surveys are based on photographic data obtained using the Oschin Schmidt Telescope on Palomar Mountain and the UK Schmidt Telescope. The plates were processed into the present compressed digital form with the permission of these institutions.
Akerlof, C. W., et al. 2003, PASP, 115, 132

Berger, E., et al. 2001, ApJ, 556, 556

2003, Nature, 426, 154

Bertin, E., \& Arnouts, S. 1996, A\&AS, 117, 393

Bessell, M. S. 1979, PASP, 91, 589

Cohen, M., Wheaton, W. A., \& Megeath, S. T. 2003, AJ, 126, 1090

Fitzpatrick, E. L., \& Massa, D. 1988, ApJ, 328, 734

Fruchter, A., \& Hook, R. N. 1997, Proc. SPIE, 3164, 120

\section{REFERENCES}

Galama, T. J., Wijers, R. A. M. J., Bremer, M., Groot, P. J., Strom, R. G., Kouveliotou, C., \& van Paradijs, J. 1998, ApJ, 500, L97

Gotz, D., \& Mereghetti, S. 2005, GCN Circ. 3329, http:/gcn.gsfc.nasa.gov/ $\mathrm{gcn} / \mathrm{gcn} 3 / 3329 . \mathrm{gcn} 3$

Gotz, D., Mereghetti, S., Mowlavi, N., Shaw, S., Beck, M., \& Borkowski, J. 2005, GCN Circ. 3323, http://gcn.gsfc.nasa.gov/gen/gen3/3323.gen3

Granot, J., \& Sari, R. 2002, ApJ, 568, 820

Guidorzi, C., et al. 2005, ApJ, 630, L121 
Henden, A. 2005, GCN Circ. 3454, http://gcn.gsfc.nasa.gov/gcn/gcn3/3454.gcn3 Huang, K. Y., et al. 2005, ApJ, 628, L93

Hurkett, C., Page, K., Osborne, J. P., Zhang, B., Kennea, J., Burrows, D. N., \& Gehrels, N. 2005, GCN Circ. 3374, http://gen.gsfc.nasa.gov/gen/gen3/3374.gcn3 Jakobsson, P., et al. 2003, A\&A, 408, 941

Landsman, W. B. 1995, in ASP Conf. Ser. 77, Astronomical Data Analysis Software and Systems IV, ed. R. A. Shaw, H. E. Payne, \& J. J. E. Hayes (San Francisco: ASP), 437

Mészáros, P., \& Rees, M. J. 1997, ApJ, 476, 232

Mészáros, P., Rees, M. J., \& Wijers, R. A. M. J. 1998, ApJ, 499, 301

Nakar, E., \& Piran, T. 2003, ApJ, 598, 400

Panaitescu, A., \& Kumar, P. 2002, ApJ, 571, 779

Panaitescu, A., \& Mészáros, P. 1999, ApJ, 526, 707

Piran, T. 2005, Rev. Mod. Phys., 76, 1143

Prochaska, J. X., Ellison, S., Foley, R. J., Bloom, J. S., \& Chen, H.-W. 2005, GCN Circ. 3332, http://gcn.gsfc.nasa.gov/gen/gen $3 / 3332$.gcn3
Reichart, D. E. 2001, ApJ, 553, 235

Sari, R., \& Piran, T. 1999, ApJ, 520, 641

Sari, R., Piran, T., \& Halpern, J. P. 1999, ApJ, 519, L17

Sari, R., Piran, T., \& Narayan, R. 1998, ApJ, 497, L17

Sato, R., Kawai, N., Suzuki, M., Yatsu, Y., Kataoka, J., Takagi, R., Yanagisawa, K., \& Yamaoka, H. 2003, ApJ, 599, L9

Schlegel, D. J., Finkbeiner, D. P., \& Davis, M. 1998, ApJ, 500, 525

Stetson, P. B. 1987, PASP, 99, 191

Willis, A. J. 1991, in IAU Symp. 143, Wolf-Rayet Stars and Interrelations with Other Massive Stars in Galaxies, ed. K. van der Hucht \& B. Hidayat (Dordrecht: Kluwer), 265

Yost, S. A., Harrison, F. A., Sari, R., \& Frail, D. A. 2003, ApJ, 597, 459

Yost, S. A., Swan, H., Schaefer, B. A., \& Alatalo, K. 2005, GCN Circ. 3322, http://gen.gsfc.nasa.gov/gen/gen3/3322.gen3 